\title{
Supporting Information: Ten-Port Unitary Optical Processor on a Silicon Photonic Chip
}

Rui Tang*, Ryota Tanomura, Takuo Tanemura*, and Yoshiaki Nakano

Department of Electrical Engineering and Information Systems, The University of Tokyo, Bunkyo-ku, Tokyo 1138656, Japan

* rui.tang@hotaka.t.u-tokyo.ac.jp; tanemura@ee.t.u-tokyo.ac.jp

\section{Contents:}

- Number of target matrices in the numerical analysis

- Numerical analysis of the fabricated 10-port UOP

- Experimental result of the fabricated 10-port UOP 


\section{Number of target matrices in the numerical analysis}

Figure $\mathrm{S} 1$ shows the average MSE after optimizing a 16-port UOP $(M=16, L=100 \mu \mathrm{m}, G=$ $300 \mathrm{~nm}, \Delta \Phi=0.01 \mathrm{rad}$ ) when different numbers of target matrices are used. The error bar represents the standard deviation. No obvious changes in the MSE can be observed when increasing the number of tested target matrices from 100 to 10000 . Therefore, we conclude that the device performance can be correctly evaluated in the numerical analysis by testing 100 target matrices.
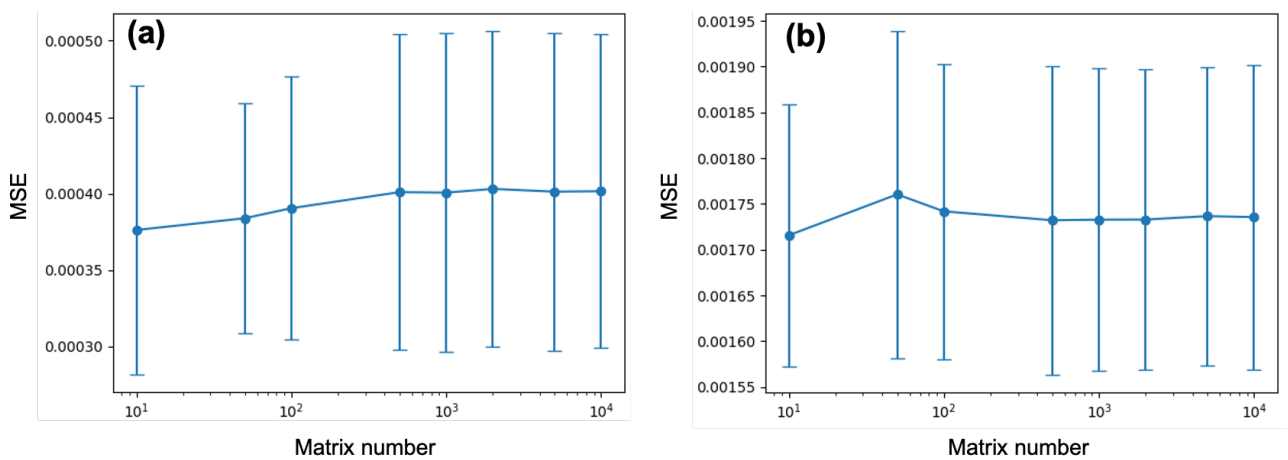

Fig. S1. Average MSE as a function of the number of tested target matrices after optimizing a 16-port UOP $(M=16, L$ $=100 \mu \mathrm{m}, G=300 \mathrm{~nm}, \Delta \Phi=0.01 \mathrm{rad}$ ). (a) For the TE polarization. (b) For the TM polarization.

\section{Numerical analysis of the fabricated 10-port UOP}

Figure S2(a) shows the calculated MSE of the fabricated 10-port UOP as a function of $\Delta \Phi$. As expected, when $\Delta \Phi$ decreases, the MSE decreases and the difference between the two polarizations becomes more obvious. Figure S2(b) shows the calculated extinction ratio after implementing the two permutation matrices used in the experiment, where the weight sum of desired power transmittances is used as the figure of merit. Note that the port-dependent coupling loss is not considered in the calculation, while in the real device, it has posed a negative impact to the device performance.
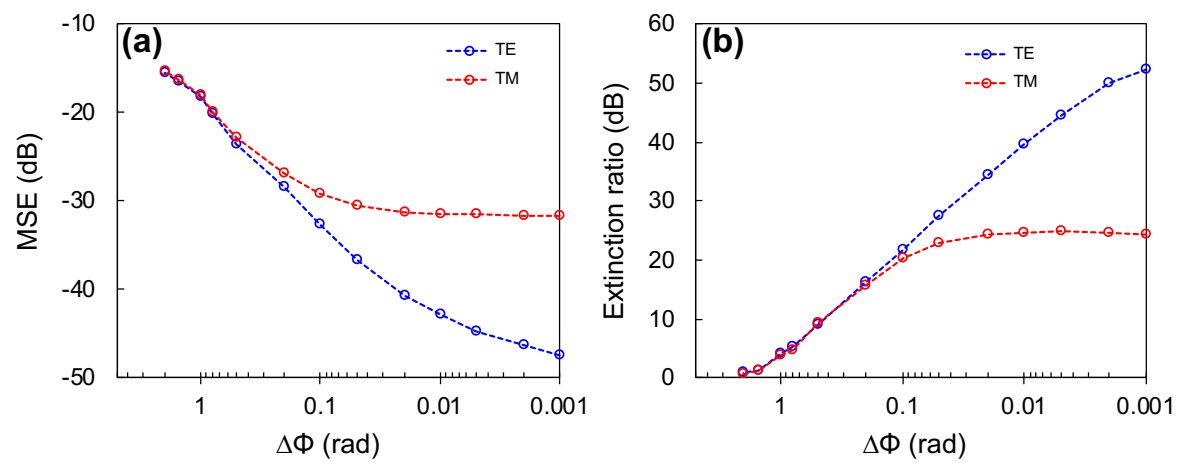

Fig. S2. Calculated device performance of the fabricated 10-port UOP as a function of $\Delta \Phi$. (a) Calculated MSE. (b) Calculated extinction ratio after implementing the two permutation matrices used in the experiment. The port-dependent coupling loss is not considered in the calculation. 


\section{Experimental result of the fabricated 10-port UOP}

Figure S3(a-b) show the measured transmittance matrices for the TE and TM polarization, respectively, before optimizing the phase shifters. Figure S3(c-f) show the results of implementing two permutation matrices for the two polarizations. Due to the large coupling loss of Out-3 port, this port cannot be optimized as desired.

(a)
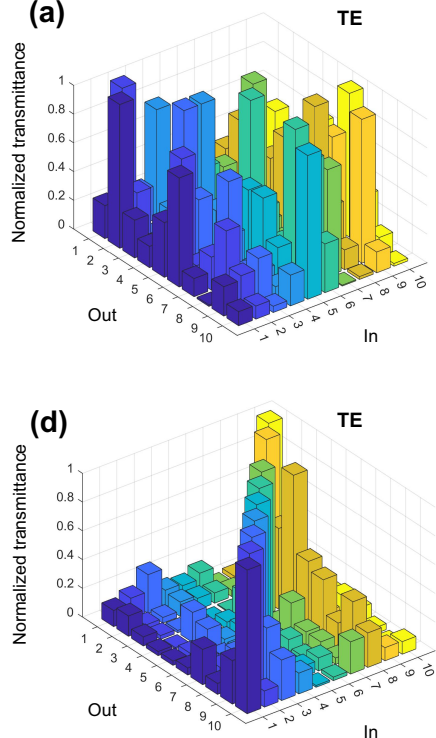

(b)

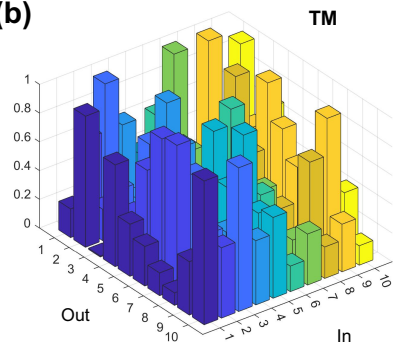

(e)

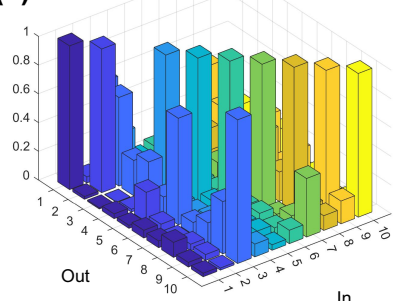

(c)

TE
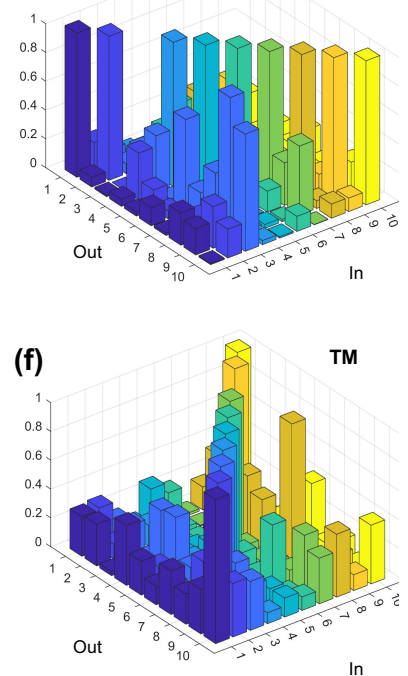

Fig. S3. Measured intensity transmittance matrices of the 10-port UOP. (a) Before optimizing the UOP, for the TE polarization. (b) Before optimizing the UOP, for the TM polarization. (c) After optimizing the UOP to implement a permutation matrix, for the TE polarization. (d) After optimizing the UOP to implement another permutation matrix, for the TE polarization. (e) After optimizing the UOP to implement a permutation matrix, for the TM polarization. (f) After optimizing the UOP to implement another permutation matrix, for the TM polarization. 